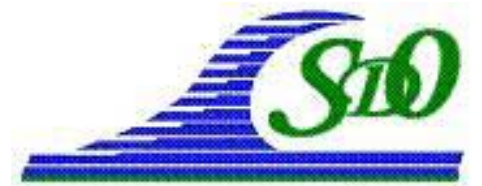

XI ${ }^{\text {èmes }}$ Journées Nationales Génie Côtier - Génie Civil

Les Sables d'Olonne, 22-25 juin 2010

DOI:10.5150/jngcgc.2010.008-C @ Editions Paralia CFL

disponible en ligne - http://www.paralia.fr - available online

\title{
Vagues sur la côte aquitaine : régionalisation dynamique de 1958 à 2002
}

\author{
Elodie CHARLES ${ }^{1,2}$, Déborah IDIER ${ }^{1}$, Rodrigo PEDREROS ${ }^{1}$, \\ Gonéri LE COZANNET ${ }^{1}$, Fabrice ARDHUIN ${ }^{3}$, \\ Cyril MALLET ${ }^{1}$, Serge PLANTON $^{2}$
}

1. BRGM, Service RNSC, 3 av. C. Guillemin, BP 6009, 45060 Orléans cedex 2, France. e.charles@brgm.fr;d.idier@brgm.fr;r.pedreros@brgm.fr;g.lecozannet@brgm.fr ; c.mallet@brgm.fr

2. Centre National de Recherches Météorologiques, 42 av. G. Coriolis, 31057 Toulouse cedex 1, France. serge.planton@meteo.fr

3 Ifremer, Centre de Brest, 29280 Plouzané, France.

fabrice.ardhuin@ifremer.fr

\section{Résumé :}

Un système de modélisation de vagues, forcé uniquement par des champs de vent a été mis en place avec le code WAVEWATCH III $^{\mathrm{TM}}$ sur une période de 44 ans (1958 à 2002) en vue d'étudier l'impact de la variabilité climatique passée sur les états de mer et sur l'érosion de la côte aquitaine. Les emboîtements hauturiers forcés par les champs de vent de la réanalyse ERA-40 ont été calibrés sur la période 1998-2002 sur 8 points de mesures. Les résultats sur 44 ans ont ensuite été validés sur 11 bouées (mesures disponibles à partir de 1980), et comparés aux modélisations existantes.

Mots-clés :

Modélisation - Vagues - Atlantique Nord - Côte aquitaine - WAVEWATCH III ERA-40

\begin{abstract}
:
A wave prediction model, forced by wind only and based on WAVEWATCH III ${ }^{\mathrm{TM}}$, was developed on a 44-year period (1958 to 2002) with the intent of studying past climate variability impact on sea states and morphological changes on the Aquitanian Coast. Deep-sea nests, forced by ERA-40 reanalysis wind fields, were calibrated on 8 buoys from 1998 to 2002. Then, the 44-year hindcast was validated against 11 buoys (measures starting from 1980) and compared to other existing simulations.
\end{abstract}

Keywords:

Modelling - Waves - North Atlantic - Aquitaine coast - WAVEWATCH III - ERA-40 


\section{Introduction}

Les littoraux sableux font partie des zones vulnérables aux changements climatiques. Leur évolution morphologique dépend de plusieurs facteurs hydrodynamiques dont les vagues sont un des principaux. Or, dans le contexte du changement climatique, les climats de vagues sont susceptibles d'être modifiés. A l'échelle du bassin océanique, des études prévoient par exemple une migration des trajectoires des tempêtes vers les pôles (YIN, 2005). Plus localement, en Mer du Nord GRABEMANN \& WEISSE (2008) montrent une augmentation probable de 5 à $8 \%$ des hauteurs des états de mer extrêmes d'ici la fin du $21^{\mathrm{e}}$ siècle, et ce à l'aide de modèles de vagues emboîtés descendant à une résolution de 5,5 km.

Le présent travail s'inscrit dans l'étude, par modélisation, des changements morphologiques passés et futurs de la côte aquitaine à l'échelle du siècle, face aux changements climatiques. La côte aquitaine (240 km de linéaire côtier), soumise à un régime méso-tidal, est fortement contrôlée par les vagues en provenance de l'Atlantique (figure 1). Les changements morphologiques s'effectuent sur un large spectre d'échelles spatio-temporelles, allant de quelques jours à plusieurs décennies. Il est donc nécessaire de connaître les caractéristiques des vagues à une haute résolution spatiale à proximité de la côte (de l'ordre du kilomètre), sur une durée pluri-décennale et ce, aussi bien sur le passé que le futur.

En termes de connaissance des climats de vague passés, il existe des mesures de vagues (bouée Gascogne, CNRM, depuis 1998 ; bouée Biscarrosse, CELM, depuis 1980 ; bouées CANDHIS, depuis 1985) mais elles sont spatialement isolées et la plupart ne concerne qu'une période récente. Le projet ANEMOC (BENOIT \& LAFON, 2004) a permis d'améliorer la connaissance des climats de vagues sur nos côtes par modélisation numérique, pour la période 1979-2002, en s'appuyant sur une validation limitée à quatre points de mesure. La réanalyse ERA-40 du CEPMMT (1957-2002) a permis de reconstituer le champ de vagues sur une plus grande période, mais la résolution spatiale $\left(\mathrm{dx}=1.125^{\circ}\right)$ est insuffisante pour une analyse des vagues côtières. Ainsi, les données actuelles ne permettent pas une caractérisation des vagues avec une résolution et sur une période suffisantes pour l'étude de l'évolution morphologique de la côte aquitaine. Concernant les climats de vagues futurs, aucune information n'est pour l'instant disponible sur le domaine littoral français. Les seules données disponibles pour le futur proviennent d'expériences de projection et concernent les variables climatiques. Il apparaît donc nécessaire de mettre en place un modèle permettant de générer les vagues à partir des prévisions de vent et de les propager jusqu'à la côte, jusqu'à une résolution kilométrique. Le développement de cette approche est d'autant plus opportun que d'importantes avancées scientifiques ont été réalisées dans la modélisation des vagues depuis les dernières simulations. Dans ce contexte, cette communication présente le système de génération et de propagation des vagues composé de trois emboîtements forcés par le vent issu de la réanalyse ERA-40, puis la calibration et la 


\section{XI $I^{\text {èmes }}$ Journées Nationales Génie Côtier - Génie Civil}

Les Sables d'Olonne, 22-25 juin 2010

validation des deux emboîtements hauturiers basés sur le modèle de vagues WAVEWATCH III qui permettront de forcer le dernier emboîtement côtier.

\section{Présentation du système de modélisation de vagues}

Afin de couvrir les zones de génération des vagues impactant le littoral aquitain tout en obtenant des résultats à la résolution kilométrique le long de la côte aquitaine, un emboitement sur 3 niveaux est mis en place, allant d'une emprise océanique à une emprise côtière (figure 1). Le premier rang R1 couvre l'Océan Atlantique Nord $\left(\mathrm{dx}=0.5^{\circ}\right)$. Le rang $\mathrm{R} 2$ couvre le Golfe de Gascogne $\left(\mathrm{dx}=0.1^{\circ}\right)$. Le rang $\mathrm{R} 3$ est resserré sur la côte aquitaine avec une résolution kilométrique.

Le code spectral de génération-propagation des vagues WAVEWATCH III (WW3) (TOLMAN 2008) est utilisé avec les paramétrages d'ARDHUIN et al. (2010) pour les rangs hauturiers $\mathrm{R} 1$ et $\mathrm{R} 2$. Le spectre est discrétisé en 24 directions et 32 fréquences.

Les champs de vent sont l'unique forçage de ce système emboîté. Les deux rangs hauturiers sont calibrés et validés sur le passé avec le vent à $10 \mathrm{~m}$ issu de la réanalyse ERA-40 du CEPMMT, disponible sur la zone de génération des vagues de 1958 à 2002 toutes les 6 heures, avec une résolution spatiale de $1.125^{\circ}$.

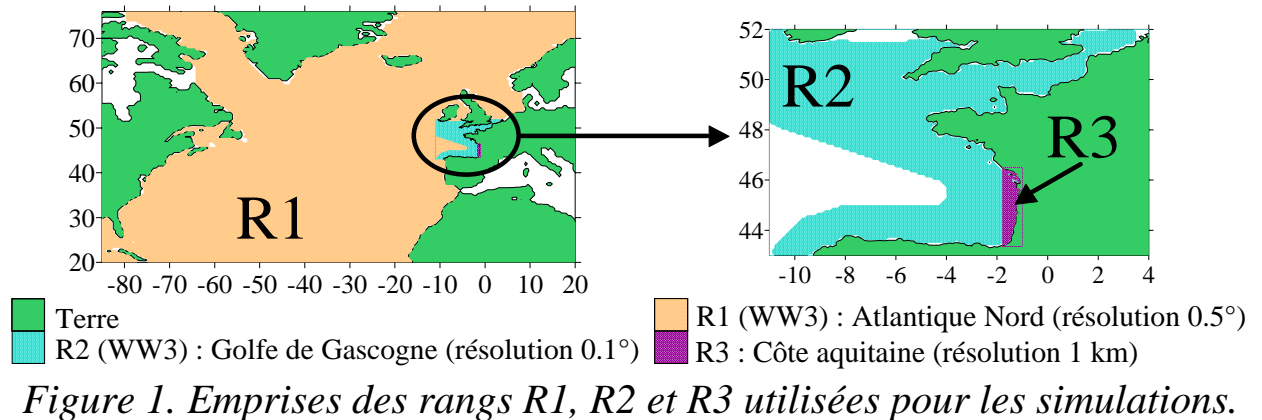

\section{Calibration des emboîtements $R 1$ et $R 2$}

Les rangs hauturiers R1 et R2 sont calibrés sur la période août 1998 - août 2002 en utilisant les mesures de la bouée Gascogne (CNRM), des bouées de la base CANDHIS et de la bouée Biscarrosse (CELM) (figure 2a). Etant donnée la position stratégique de la bouée Gascogne (représentative des conditions de vagues au large du site d'étude), la calibration est faite en priorité sur cette bouée, puis sur les bouées de la façade atlantique, et enfin sur les bouées en Manche.

La première étape est de comparer le vent observé au vent utilisé dans le modèle de vagues car dans des conditions de vent constant, l'erreur relative de la hauteur de vague est deux fois plus grande que l'erreur relative de la vitesse du vent (JANSSEN et al. 2001). La figure $2 \mathrm{~b}$ met en évidence que les vitesses du vent ERA-40 sont sousestimées au niveau de la bouée Gascogne (le vent ERA-40 étant donné à une hauteur de $10 \mathrm{~m}$, il est ramené à la hauteur de l'anémomètre, $3 \mathrm{~m}$, en faisant l'hypothèse d'un profil de vent logarithmique). Il est donc nécessaire d'appliquer une correction soit sur le vent 
en entrée, soit sur les vagues en sortie du modèle. Pour cette étude, le choix s'est porté sur une correction du vent en entrée puisque le nombre limité de sites de mesure ne permet pas de faire une correction spatiale fine des vagues a posteriori.

Cette correction se fait dans le modèle WW3 avec le paramètre ZWND (hauteur du vent en entrée du modèle). Elle consiste à modifier la hauteur du vent donné en entrée afin de faire varier son intensité et tient compte, notamment, de la rugosité de la surface de l'eau liée aux conditions de vagues locales. Ainsi, des valeurs de ZWND comprises entre $2 \mathrm{~m}$ et $10 \mathrm{~m}$ sont utilisées dans WW3. Les résultats obtenus en termes de hauteur, période et direction des vagues sont ensuite comparés aux mesures pour estimer la valeur optimale de ZWND.
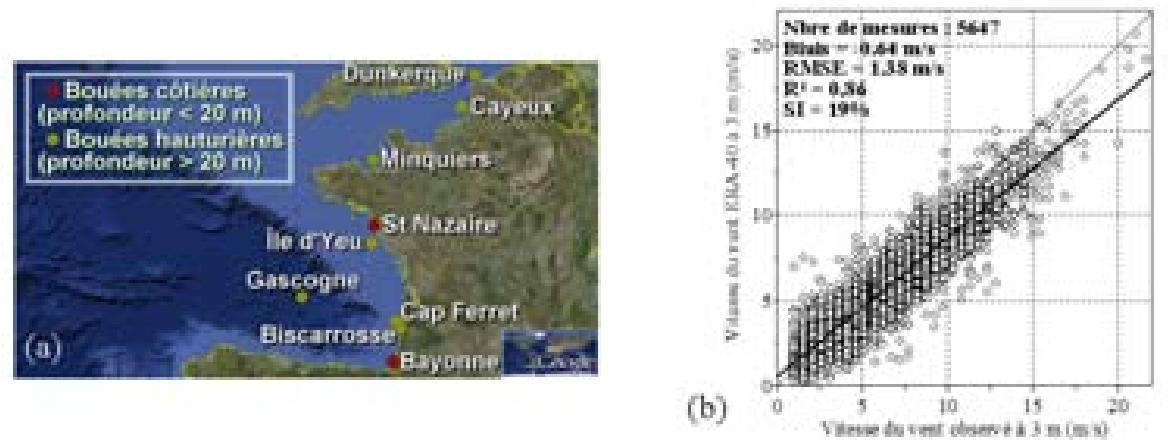

Figure 2. (a) Bouées utilisées pour la calibration et la validation des rangs $R 1$ et $R 2$, (b) Comparaison de la vitesse du vent simulée par ERA-40 (ramenée à $3 \mathrm{~m}$ ) à la vitesse du vent observée à $3 \mathrm{~m}$ à la bouée Gascogne $\left(45.201^{\circ} \mathrm{N}, 5^{\circ} \mathrm{O}\right)$.

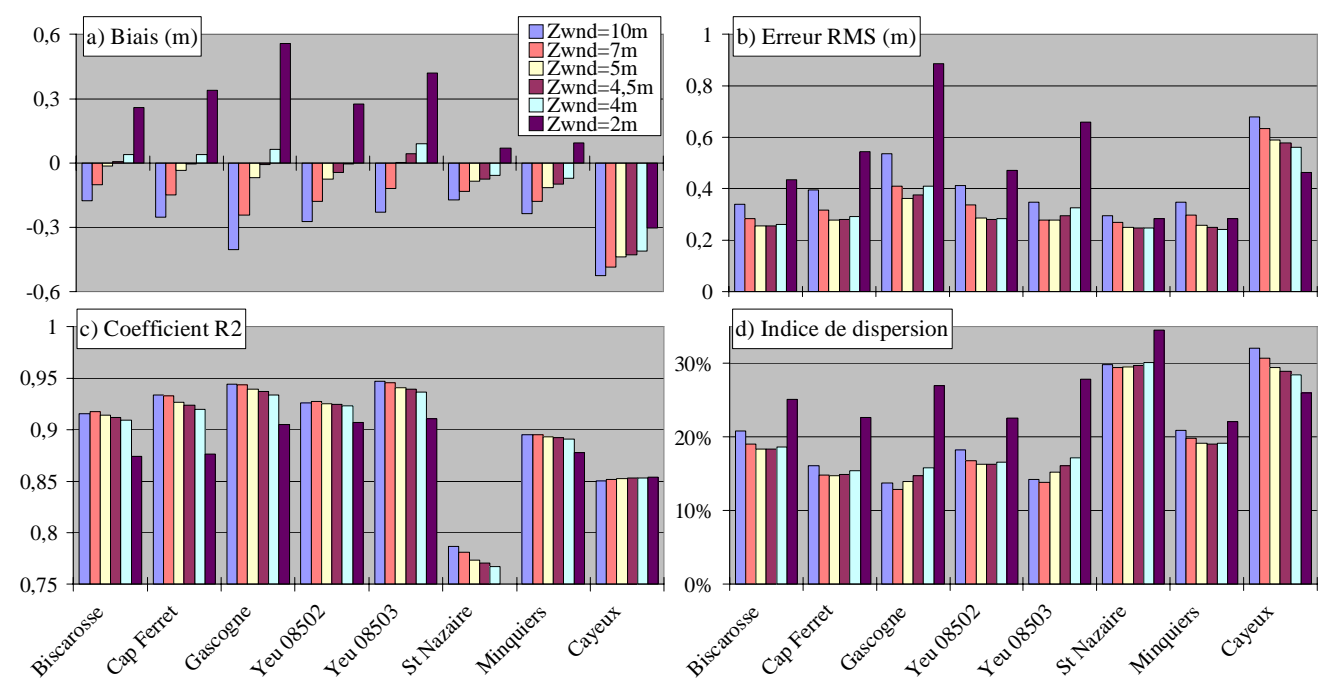

Figure 3. Comparaison des hauteurs de vagues mesurées par 8 bouées entre 1998 et 2002, et des hauteurs de vagues simulées par WW3 pour différentes valeurs de ZWND :

(a) Biais, (b) erreur RMS, (c) coefficient de corrélation $R^{2}$, (d) indice de dispersion.

Afin de confronter mesures et simulations sur une échelle de temps comparable, une étape préalable a consisté à enlever les mesures aberrantes (par contrôle automatique et 


\section{XI $I^{\text {èmes }}$ Journées Nationales Génie Côtier - Génie Civil}

Les Sables d'Olonne, 22-25 juin 2010

manuel), puis à calculer les moyennes quadratiques (pour les hauteurs et périodes) et linéaires (pour les directions) des deux types de données sur des fenêtres temporelles de 3 heures.

Les statistiques relatives aux hauteurs de vagues (figure 3) montrent qu'on obtient un coefficient de corrélation similaire pour des valeurs de ZWND comprises entre 4 et $10 \mathrm{~m}$, qui se dégrade fortement à $2 \mathrm{~m}$. Pour une valeur de ZWND égale à 4,5 $\mathrm{m}$, on obtient un biais quasi-nul, une erreur RMS entre 25 et $37 \mathrm{~cm}$, et un indice de dispersion entre 14 et $18 \%$ pour les bouées hauturières situées sur la façade Atlantique.

Concernant la période moyenne T02, le coefficient de corrélation varie peu pour des valeurs de ZWND comprises entre 4 et $10 \mathrm{~m}$ (environ 0,82 pour la bouée Gascogne, 0,70 pour le Cap Ferret, 0,45 pour Biscarrosse). Le biais et l'erreur RMS sont optimisés au niveau des bouées Gascogne (resp. $-0,17$ s et 0,77 s) et Minquiers (resp. +0,05 s et $1,07 \mathrm{~s}$ ) pour une valeur de ZWND égale à $4,5 \mathrm{~m}$. Cependant, au niveau des autres bouées de la façade Atlantique, les biais sont compris entre $+0,31$ et $+0,81$ s pour le vent ERA-40 d' origine, et augmentent d'environ 0,4 s quand on abaisse le vent à 4,5 m.

Concernant les directions pics, la comparaison avec les bouées directionnelles donne une erreur RMS d'environ $25^{\circ}$ à Yeu et $32^{\circ}$ aux Minquiers, très peu dépendante de la hauteur du vent. Quant au biais, il passe de $-5,3^{\circ}$ à $-1,4^{\circ}$ à Yeu et de $1,9^{\circ}$ à $0,2^{\circ}$ aux Minquiers lorsqu' on abaisse la hauteur du vent de $10 \mathrm{~m}$ à $2 \mathrm{~m}$.

Le choix de la valeur de ZWND égale à 4,5 m est donc un bon compromis compte tenu des résultats en termes de hauteur, période et direction au niveau de la bouée Gascogne et des autres bouées de la façade atlantique.

\section{Validation des emboîtements $R 1$ et $R 2$ sur la période 1980-2002}

Une fois les emboîtements R1 et R2 calibrés, la période 1958-2002 est simulée avec les champs de vent ERA-40. La qualité de la réanalyse ERA-40 évolue au cours du temps en raison d'une augmentation considérable des données d'assimilation sur les années récentes (très peu de données satellitaires avant 1988). La qualité des vagues forcées par ce vent évolue donc également entre 1958 et 2002, et il convient de rester critique quant aux vagues modélisées avant 1980 pour lesquelles nous n'avons aucune mesure locale. Dans un premier temps, les caractéristiques des vagues sont comparées à des mesures réparties sur la période 1980-2002 selon la méthode décrite pour la calibration (tableau 1). Les résultats en termes de hauteurs et directions sont très satisfaisants compte-tenu de la résolution spatiale et angulaire. Concernant la période moyenne, le biais est satisfaisant au niveau de la bouée Gascogne, mais il est d'environ $+1 \mathrm{~s}$ au niveau des autres bouées.

Du fait de sa longue période de déploiement (1980-...), la bouée Biscarrosse a été utilisée dans de précédentes études pour caractériser la climatologie des vagues du Golfe de Gascogne (BUTEL et al., 2002) et leur variabilité interannuelle (DUPUIS et al., 2006). Il est donc intéressant de regarder plus en détail les résultats du modèle au 
niveau de cette bouée déployée face à une plage de sable rectiligne, représentative d'une grande partie des plages linéaires sableuses de la côte aquitaine. Les statistiques sur la période 1980-2002 (figure 4a) indiquent une bonne corrélation entre les mesures et les simulations, malgré une baisse de performance par rapport à la période de calibration (figure 4b), notamment au niveau du biais qui passe de 0 à $10 \mathrm{~cm}$. Cette différence peut être attribuée partiellement à la qualité hétérogène des vents de la réanalyse ERA-40 (avant/après 1988), et aux incertitudes liées à la mesure plus fortes dans le passé.

Tableau 1. Résultats statistiques de la comparaison de la hauteur, période et direction des vagues simulées et des vagues mesurées.

\begin{tabular}{|c|c|c|c|c|c|c|c|c|c|c|}
\hline & \multicolumn{4}{|c|}{ Hauteur significative } & \multicolumn{4}{|c|}{ Période moyenne T02 } & \multicolumn{2}{|c|}{ Direction pic } \\
\hline & Biais & $\begin{array}{l}\text { Erreur } \\
\text { RMS }\end{array}$ & $R^{2}$ & $\begin{array}{l}\text { Indice de } \\
\text { dispersion }\end{array}$ & Biais & $\begin{array}{l}\text { Erreur } \\
\text { RMS }\end{array}$ & $R^{2}$ & $\begin{array}{l}\text { Indice de } \\
\text { dispersion }\end{array}$ & Biais & $\begin{array}{l}\text { Erreur } \\
\text { RMS }\end{array}$ \\
\hline $\begin{array}{l}\text { Bouée Bayonne } \\
\text { [1989-1992] }\end{array}$ & $-10 \mathrm{~cm}$ & $39 \mathrm{~cm}$ & 0,87 & $23 \%$ & $1,27 s$ & $1,85 \mathrm{~s}$ & 0,66 & $19 \%$ & - & - \\
\hline $\begin{array}{l}\text { Bouée Biscarrosse } \\
\text { [1980-2000] }\end{array}$ & $10 \mathrm{~cm}$ & $35 \mathrm{~cm}$ & 0,87 & $24 \%$ & $0,90 s$ & $1,71 \mathrm{~s}$ & 0,56 & $22 \%$ & - & - \\
\hline $\begin{array}{l}\text { Bouée Cap Ferret } \\
\text { [2001-2002] }\end{array}$ & $0 \mathrm{~cm}$ & $28 \mathrm{~cm}$ & 0,92 & $15 \%$ & $0,84 s$ & $1,38 \mathrm{~s}$ & 0,70 & $16 \%$ & - & - \\
\hline $\begin{array}{l}\text { Bouée Gascogne } \\
\text { [1998-2002] }\end{array}$ & $-1 \mathrm{~cm}$ & $38 \mathrm{~cm}$ & 0,94 & $15 \%$ & $-0,17 s$ & $0,77 s$ & 0,82 & $10 \%$ & - & - \\
\hline $\begin{array}{l}\text { Bouée Yeu } 08501 \\
{[1992-1998]}\end{array}$ & $-9 \mathrm{~cm}$ & $32 \mathrm{~cm}$ & 0,93 & $16 \%$ & $0,62 \mathrm{~s}$ & $1,24 \mathrm{~s}$ & 0,71 & $17 \%$ & - & - \\
\hline $\begin{array}{l}\text { Bouée Yeи } 08502 \\
\text { [1998-2000] }\end{array}$ & $-4 \mathrm{~cm}$ & $28 \mathrm{~cm}$ & 0,92 & $16 \%$ & $0,69 s$ & $1,14 s$ & 0,70 & $15 \%$ & $-3^{\circ}$ & $24^{\circ}$ \\
\hline $\begin{array}{l}\text { Bouée Yeи } 08503 \\
\text { [2000-2002] }\end{array}$ & $4 \mathrm{~cm}$ & $30 \mathrm{~cm}$ & 0,94 & $16 \%$ & $0,80 s$ & $1,35 \mathrm{~s}$ & 0,71 & $17 \%$ & - & - \\
\hline $\begin{array}{l}\text { Bouée St Nazaire } \\
\text { [1999-2002] }\end{array}$ & $-7 \mathrm{~cm}$ & $25 \mathrm{~cm}$ & 0,77 & $30 \%$ & $1,14 \mathrm{~s}$ & $1,89 \mathrm{~s}$ & 0,36 & $33 \%$ & - & - \\
\hline $\begin{array}{l}\text { Bouée Minquiers } \\
\text { [1992-2000] }\end{array}$ & $-9 \mathrm{~cm}$ & $26 \mathrm{~cm}$ & 0,88 & $20 \%$ & $0,10 \mathrm{~s}$ & $1,13 \mathrm{~s}$ & 0,55 & $21 \%$ & $0^{\circ}$ & $36^{\circ}$ \\
\hline $\begin{array}{l}\text { Bouée Cayeux } \\
\text { [1999-2001] }\end{array}$ & $-43 \mathrm{~cm}$ & $58 \mathrm{~cm}$ & 0,85 & $29 \%$ & $-0,16 \mathrm{~s}$ & $1,03 s$ & 0,16 & $23 \%$ & - & - \\
\hline $\begin{array}{l}\text { Bouée Dunkerque } \\
\text { [1988-1996] }\end{array}$ & $-33 \mathrm{~cm}$ & $45 \mathrm{~cm}$ & 0,76 & $31 \%$ & $-0,40 s$ & $0,79 s$ & 0,23 & $17 \%$ & - & - \\
\hline
\end{tabular}

Dans un second temps, nous comparons les performances des rangs hauturiers aux performances d'autres simulations: les vagues issues de la réanalyse ERA-40 et ANEMOC. Comparées aux mesures de la bouée Gascogne toutes les 6 heures, les hauteurs de vagues simulées par les emboitements hauturiers (biais: $0 \mathrm{~cm}$; Erreur RMS : $39 \mathrm{~cm} ; \mathrm{R}^{2}: 0.93$; indice de dispersion : 15\%) sont de bien meilleure qualité que les hauteurs de vagues issues de la réanalyse ERA-40 (biais : -34 cm ; Erreur RMS : 55 $\mathrm{cm} ; \mathrm{R}^{2}: 0.93$; indice de dispersion : 17\%). Plus spécifique aux côtes françaises, le modèle mis en place avec le code TOMAWAC pour créer la base de données ANEMOC (BENOIT \& LAFON, 2004) a été calibré sur la période 1999-2000 sur 


\section{XI $I^{\text {èmes }}$ Journées Nationales Génie Côtier - Génie Civil}

Les Sables d'Olonne, 22-25 juin 2010

quatre bouées CANDHIS. La comparaison des hauteurs de vagues mesurées à heure fixe sur la période 1999-2000 aux hauteurs de vagues simulées sur le rang R2 (dx=10 $\mathrm{km}$ ) donne une erreur RMS de $24 \mathrm{~cm}$ à l'île d'Yeu et aux Minquiers, soit plus petite que celles obtenues sur le rang océanique d'ANEMOC $\left(\mathrm{dx}=1^{\circ}\right.$ au large, $20 \mathrm{~km}$ le long des côtes) sur la même période (resp. $37 \mathrm{~cm}$ et $33 \mathrm{~cm}$ ).

Les rangs $\mathrm{R} 1$ et $\mathrm{R} 2$ mis en place donnent donc des résultats de meilleure qualité que les modèles précédents en termes de hauteurs de vague. Ils peuvent donc être utilisés pour forcer le rang R3, et pour des études limitées au Golfe de Gascogne, en tenant compte des erreurs observées sur les périodes moyennes.
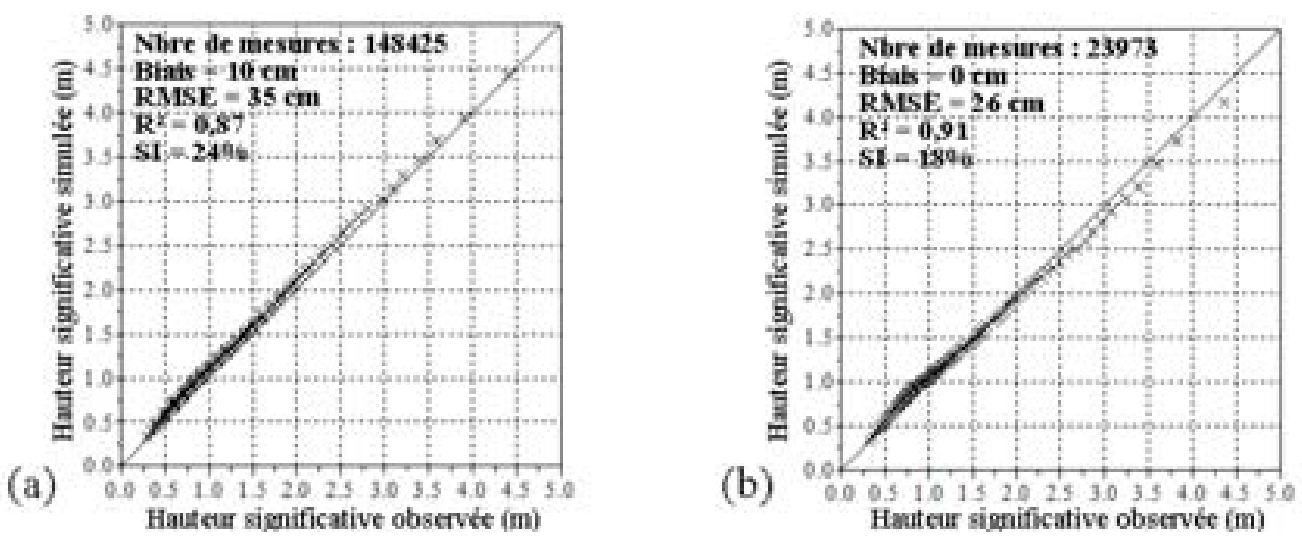

Figure 4. Comparaison quantile-quantile des hauteurs de vagues observées et simulées

au niveau de la bouée Biscarrosse (a) de 1980 à 2002, (b) de 1998 à 2002.

\section{Conclusion et perspectives}

Un système de modélisation des vagues le long de la côte aquitaine a été mis en place. Les premiers résultats sont satisfaisants sur la période 1998-2002 : dans le Golfe de Gascogne, ils sont de meilleure qualité en termes de hauteurs de vagues que les simulations existantes. La comparaison sur une longue durée (1980-2002) nous indique une possible diminution de la qualité des résultats pour les périodes plus anciennes, qui reste néanmoins acceptable. Une étude plus fine de la zone côtière sera possible par la mise en place d'un dernier emboîtement côtier. Des simulations seront alors réalisées sur le passé et le futur afin d'évaluer l'impact potentiel des modifications des climats de vagues dues aux changements climatiques et ce, sur l'évolution morphologique de la côte aquitaine.

\section{Remerciements :}

Ce travail a été réalisé dans le cadre d'une thèse BRGM-CNRM, AXA Research Fund, et co-encadrée par P. Delecluse et D. Idier. Les auteurs remercient l'équipe ERA-40, A. Braun (CNRM), A. Le Berre et X. Kergadallan (CETMEF), C. Deyts (CELM), F. Dupros et F. Boulaya (BRGM) et la région Centre (calculateur PHOEBUS). 
Thème 1 - Hydrodynamique côtière

\section{Références bibliographiques}

ARDHUIN F., ROGERS E., BABANIN A., FILIPOT J.F., MAGNE R., ROLAND A., VAN DER WESTHUYSEN A., QUEFFEULOU P., LEFEVRE J.M., AOUF L., COLLARD F. (2010). Semi-empirical dissipation source functions for ocean waves: Part I, definition, calibration and validation. J. of Physical Oceanography. doi:10.1175/2010JPO4324.1

AUBIE S., TASTET J.P. (2000). Coastal erosion, processes and rates : an historical study of the Gironde coastline, southwestern France. J. of Coastal Res., 16, 3, pp 756-767.

BENOIT M., LAFON F. (2004). A nearshore wave atlas along the coasts of France based on the numerical modeling of wave climate over 25 years. Proc. $29^{\text {th }}$ Int. Conf. on Coastal Eng. (ICCE'2004), Lisbonne (Portugal), pp 714-726.

BUTEL R., DUPUIS H., BONNETON P. (2002). Spatial variability of wave conditions on the French Atlantic coast using in-situ data. J. of Coastal Res., SI 36, pp 96-108.

DUPUIS H., MICHEL D., SOTTOLICHIO A. (2006). Wave climate evolution in the Bay of Biscay over two decades. J. of Marine Systems, 63, pp 105-114. doi:10.1016/j.jmarsys.2006.05.009

GRABEMANN I., WEISSE R. (2008). Climate change impact on extreme wave conditions in the North Sea: an ensemble study. Ocean Dynamics, 58, pp 199-212. doi:10.1007/s10236-008-0141-x

JANSSEN P.A.E.M., DOYLE J.D., BIDLOT J., HANSEN B., ISAKEN L., VITERBO P. (2001). Impact and feedback of ocean waves on the atmosphere. ECMWF Technical Memorandum No.341.

KAMPHUIS J.W. (2002). Alongshore transport of sand. 28th Coastal Engineering Conference, ASCE.

MANAUD F., L'YAVANC J., NEGRE S., TOUGERON C., TRUT G. (2001). Elaboration d'un outil de gestion prévisionnelle de la côte Aquitaine. Phase 3 : diagnostic d'évolution et recommandations. Contribution de l'IFREMER. Rapport IFREMER DEL-AR.

ROSATI J.D., WALTON T.L., BODGE K. (2002). Longshore sediment transport. Coastal Engineering Manual, Chapter III-2, Engineer Manual 1110-2-1100, U.S.A.C.E. TOLMAN H.L. (2008). A mosaic approach to wind wave modeling. Ocean Modelling, 25, pp 35-47. doi:10.1016/j.ocemod.2008.06.005

YIN J.H. (2005). A consistent poleward shift of the storm tracks in simulations of 21st century climate. Geophys. Res. Lett., 32, L18701. doi:10.1029/2005GL023684 